\title{
ULTRASOUND GUIDED THORACIC PARAVERTEBRAL BLOCK AS AN ANALGESIC TECHNIQUE FOR TRACHEOESOPHAGEAL FISTULA REPAIR : A CASE REPORT
}

\author{
Rupesh Kr. Yadav ${ }^{1 *}$, Sadichhya shah Malla', Anupama Thapa ${ }^{3}$
}

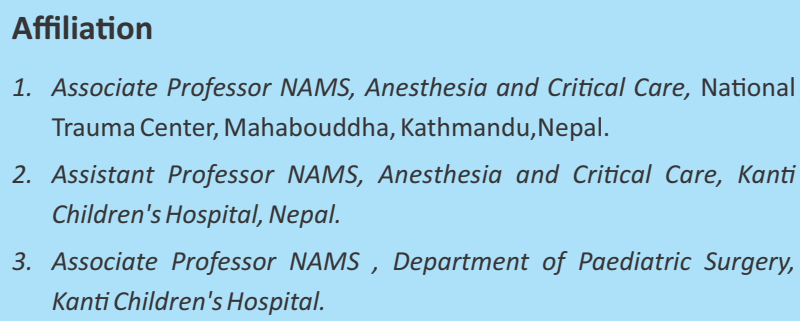

1. Associate Professor NAMS, Anesthesia and Critical Care, National Trauma Center, Mahabouddha, Kathmandu,Nepal.

2. Assistant Professor NAMS, Anesthesia and Critical Care, Kanti Children's Hospital, Nepal.

3. Associate Professor NAMS, Department of Paediatric Surgery, Kanti Children's Hospital.

\section{ARTICLE INFO}

Received : 05 January, 2021

Accepted : 26 March, 2021

Published : 15 March, 2021

(c) Authors retain copyright and grant the journal right of first publication with the work simultaneously licensed under Creative Commons Attribution License CC - BY 4.0 that allows others to share the work with an acknowledgment of the work's authorship and initial publication in this journal.

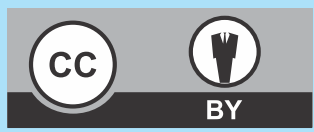

CR 40

DOI: https://doi.org/10.3126/bjhs.v6i1.37666

\author{
* Corresponding Author \\ Dr. Rupesh Kumar Yadav \\ Associate Professor \\ Department of Anesthesia and Critical Care \\ NAMS, National Trauma Center, Kathmandu, Nepal \\ Email: rupeshdoc905@gmail.com \\ ORCID ID: https://orcid.org/0000-0002-0607-6677
}

\section{Citation}

Rupesh Kr. Yadav, Sadichhya shah Malla, Anupama Thapa. Ultrasound Guided Thoracic Paravertebral block as an analgesic technique for Tracheoesophageal fistula repair : A Case Report. BJHS 2021;6(1)14. 1422-1425.

\section{ABSTRACT}

Ultrasound has now become an eye of anaesthesiologist and has encouraged for its pervasive use. Its considerable use in the perioperative period for various invasive purposes has added an extra caution to prevent unforeseen complications over blind blocks. The ultrasound-guided thoracic paravertebral block provides excellent perioperative analgesia following thoracic surgery. It can be given as a single shot or a continuous infusion technique placing a catheter. Here the local anesthetic agent is deposited in the paravertebral space from where the spinal nerve emerges which are devoid of coverings and are sensitive to local anesthetics. Even a single-shot block provides analgesia of 4-6 dermatomal levels. The use of local anesthetics using ultrasound not only adds safety measures to minimize procedural complications but also reduces the perioperative requirement of opioids. It also helps in the reduction of postoperative pulmonary complications and early extubation following thoracic surgery. We report a case series where single-shot ultrasound-guided unilateral paravertebral blocks were used as an analgesic technique for the repair of tracheoesophageal fistula in neonates. After delivering anesthesia and confirming the correct placement of the endotracheal tube the neonate was kept on the left lateral position to undergo right thoracotomy and repair of fistula. Scanning at T4 level with a linear probe was done to identify the transverse process, pleura, costotransverse ligament, intercostal muscle, and the paravertebral space where the local anesthetic agent was deposited under aseptic precautions. Tenting of pleura was the endpoint for the completion of the block. The extensive use of various regional anesthetic technique has significantly improved the postoperative outcome of the patients along with adequate perioperative pain management.

\section{KEY WORDS}

Paediatrics, paravertebral block, tracheo-esophageal fistula , ultrasound 


\section{INTRODUCTION}

Ultrasound is now being extensively used by anesthesiologist throughout the perioperative period either as a diagnostic tool to aid early diagnosis as a part of safety measures or as a pain management tool along with various regional anesthetic technique. Regional anesthesia these days are gaining popularity over the blind blocks which were practiced earlier. With the help of ultrasound the nerves are clearly visualized and the deposition of local anesthetic agent along with its spread can be seen well. The identification and confirmation of the nerves can be done using a periphereal nerve stimulators. Where-as for the plane blocks the drug distribution along the interfascial plane can be seen clearly and even if a catheter is placed for continuous infusion the ultrasound compatible catheters can be seen clearly in the ultrasound.

Ultrasound guided Thoracic Paravertebral blocks are these days being extensively used for various thoracic and upper abdominal surgeries as an important anesthetic tool for perioperative pain management either as a single shot or a continuous catheter technique. With the visualization of the paravertebral space and the drug deposition via Ultrasound, adequacy of block can be well anticipated.

Huge consumption of opioids with delay in extubation and postoperative pulmonary complication provides a tremendous challenges for anaesthesiologist in the part of pain management following major thoracic surgeries.

We would like to report few cases of right thoracotomy performed for tracheoesophageal fistula repair where modality of pain management was done using ultrasound guided single shot right sided paravertebral block.

\section{CASE 1}

4 days old child weighing $3.5 \mathrm{~kg}$, a referred case of tracheoesophageal fistula was planned for right thoracotomy and repair of fistula. During pre anesthetic checkup the routine blood counts were within normal range for age. Chest radiograph showed opacity on right upper apical zone with coiling of orogastric tube on upper pouch. On auscultation fine crepitations were heard on both lung field. Oxygen saturation at room air was $88 \%$ which improved to $92 \%$ with supplementation of oxygen via head box at the flow rate of 10 liters per minute. ECHO report showed small ASD of $2.2 \mathrm{~mm}$ size with left to right shunt and a normal PA Pressure. The patient party were counseled and consent was taken regarding anesthetic procedure. Adequate blood products were arranged. As the child was kept on NPO, fluid and calorie supplementation was given through intravenous route. On arrival to the operation theatre, file was reviewed and child confirmed. ASA standard monitoring were attached. As the intravenous line was in situ it was checked for the patency and induction was initiated with Propofol $10 \mathrm{mg}$, Fentanyl $4 \mathrm{mcg}$ and sux $6 \mathrm{mg}$ as muscle relaxant and airway was secured with $3.5 \mathrm{~mm}$ internal diameter uncuffed endo tracheal tube and fixed after auscultation at the both lung fields. The child was then turned to left lateral position and using a high frequency linear probe scanning was done at T4 level. On identifying the spinous process the probe was gradually made oblique and on further scanning transverse process and pleura was visualized. Using a 26 ' $G$ ' Quincke spinal needle the needle was inserted in an oblique in-plane view and a PMO (M-F) line was connected at the hub of the spinal needle after removing the stylet and $2.0 \mathrm{ml}$ of local anesthetic agent with a combination of $0.25 \%$ of Bupivacaine and 1\% Xylocaine with Adrenaline was deposited in the paravertebral space. Visualization of drug deposition in the paravertebral space and tenting of pleura was the end point of scanning. The child was left on the same left lateral position as the surgery was to be performed in the same position. Surgery was started and maintenance of anesthesia was done with $\mathrm{O} 2+\mathrm{ISO}+$ Vecuronium and assist controlled ventilation. Two episodes of desaturation up to $80 \%$ were noticed during intra operative period which was managed with bag mask ventilation and release of traction at the right lung around the operative field. There were no fluctuation in intraoperative hemodynamic and the surgery lasted for 90 minutes. With endotracheal tube in situ the child was transferred to surgical intensive care unit and extubation was done after gaining airway reflexes on the same day. NIPS ( Neonatal-Infant Pain Scale ) scoring system was used to assess postoperative pain hourly for first 6 hour than 2 hourly for remaining 24 hours. A score greater than 3 is an indicator of pain which was managed with intravenous Fentanyl 5 microgram and Paracetamol $20 \mathrm{mg} / \mathrm{kg}$ body weight. The child required only a single doses of intravenous Fentanyl in the first 24 hours. On the 5 th post operative day the child was given $0.5 \mathrm{ml}$ of Methylene blue via oral route to check for any anastomotic leakage. On confirmation of no leakage feeding was started on 5th postoperative day. Chest drain was taken out on the same day and was shifted to ward on 7 th post operative day. The child was discharged to home on $10^{\text {th }}$ post operative day.

\section{CASE 2}

2 days old child weighing $2.5 \mathrm{~kg}$ a diagnosed case of tracheoesophageal fistula was planned for right thoracotomy and fistula repair. During pre anesthetic check up the Chest radiograph showed coiling of orogastric tube on upper pouch and on auscultation of chest there was equal air entry with no added sounds. Saturation at room air was $92 \%$ so oxygen supplementation with a nasal prong at the rate of 2 liters per minute was supplemented which showed improvement to $99 \%$. ECHO report was normal. The 
routine biochemistry was within normal range. Intravenous anaesthesia was delivered and air way was secured with appropriate size uncuffed endotracheal tube. Ultrasound guided right sided thoracic paravertebral block was given at T4 level. $1.3 \mathrm{ml}$ volume local anaesthetics of $0.25 \%$ Bupivacaine and $1 \%$ Xylocaine with Adrenaline was deposited at the paravertebral space. Maintainenance of anaesthesia was done with $\mathrm{O} 2+\mathrm{ISO}+$ Vecuronium and assist controlled ventilation. One episode of Bradycardia was noticed intraoperatively which was managed with Intravenous Atropine and also few episodes of desaturation up to $75 \%$ were noticed which was managed with the release of traction to the lungs and bag mask ventilation. The intraoperative surgical period was around 70 minutes. The child was extubated in the operation theater and shifted to SICU. During postoperative period the child received two doses of Intravenous Fentanyl in the first 24 hours as the NIPS score was greater than 3 on assessment. Rest postoperative periods were uneventful, the child was shifted to ward on $10^{\text {th }}$ postoperative day and discharged to home on $14^{\text {th }}$ post operative day.

\section{CASE 3}

A referred case of 7 days old child weighing $3.0 \mathrm{~kg}$ who was diagnosed as Tracheoesophageal fistula was planned for Right thoracotomy and repair of fistula. During pre anesthetic checkup the Chest X-Ray showed opacity on both upper zone with coiling of orogastric tube on upper pouch . On auscultation fine crepitations were heard. SPO2 on room air was $84 \%$ which improved to $92 \%$ with Oxygen supplementation via head box. ECHO report showed ASD, VSD, PDA with left to right shunt and normal PA Pressure. Intravenous anaesthesia with endotracheal intubation and Ultrasound guided unilateral right sided thoracic paravertebral block at T4 level as a perioperative mode of analgesia technique was delivered using $1.3 \mathrm{ml}$ of local anaesthetics of $0.25 \%$ Bupivacaine and $1 \%$ Xylocaine with Adrenaline. Few episodes of desaturation were noticed intra operatively which were managed with release of traction and bag mask ventilation, two episodes of bradycardia was noticed which was managed with intravenous Atropine. Intraoperative blood transfusion was needed due to up limit of allowable blood loss and after clinical assessment. The child also required intraoperative Dopamine due to hemodynamic instability and the surgery lasted for around 110 minutes. With the tube in situ the child was transferred to SICU and planned for elective ventilation. Pain assessment was done with Hartwig score as the child was intubated and electively ventilated. During postoperative period the child received only one doses of Intravenous Fentanyl in the first 24 hours as the score was more than 7. Extubation was done on 2nd post operative day after seeing the vitals, blood reports, $A B G$ report and $C-X$ ray. On the 6 th post operative day the child was given $0.5 \mathrm{ml}$ of Methylene blue to check for any anastomotic leakage and on confirmation of no leakage feeding was started. Rest of the postoperative periods were uneventful, the child was shifted to ward on 10th postoperative day and discharged to home on 15th postoperative day.

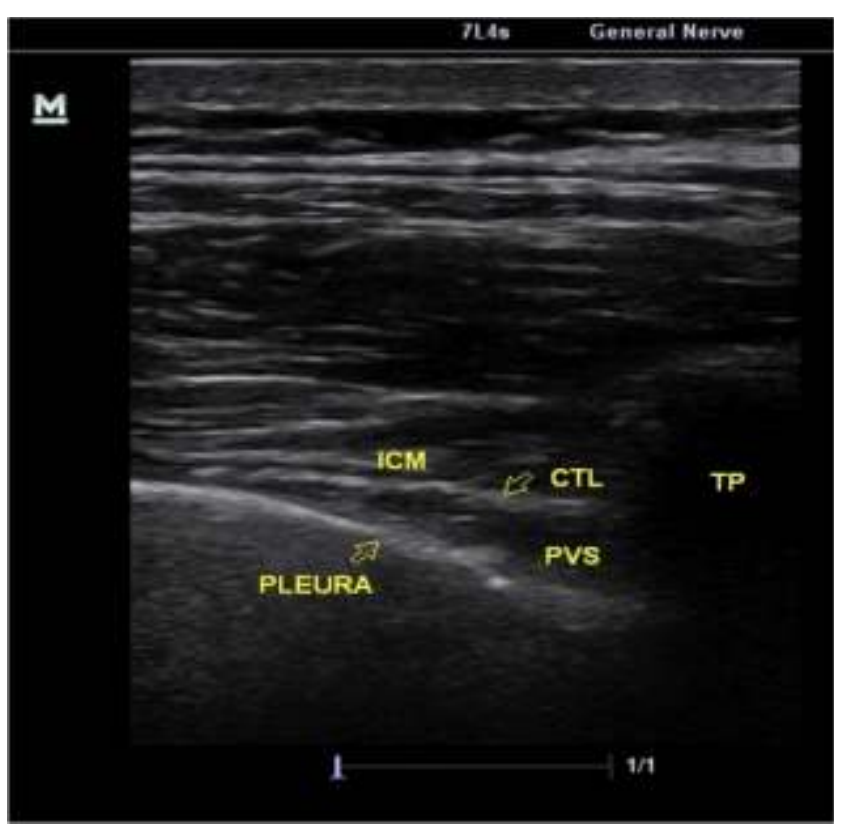

Figure 1: Ultrasound image showing Paravertebral space.

PVS-Paravertebral space, CTL- Costotransverse ligament, ICM-Intercostal muscle, TP-Transverse process

\section{DISCUSSION}

Tracheoesophageal fistula (TEF) is a rare congenital developmental anomaly affecting 1 in 2500 live births. ${ }^{1}$ In a developing country like ours, repair of TEF is still being performed through open thoracotomy with various postoperative problems as postoperative incisional pain, prolong postoperative ventilator support and pulmonary complications. To date, caudal epidural analgesia has been successfully used along with general anesthesia in neonates with TEF for pain management and reduction for the need of postoperative ventilator support. ${ }^{2}$ In a study by Kinottenbet G et al a caudal catheter was advanced to T6-T7 to supplement the general anesthesia and provide excellent postoperative analgesia without the use of opioids and to facilitate early extubation. ${ }^{3}$ Recently Ultrasound-guided thoracic paravertebral block has applied to a large number of populations including pediatrics as it is a high-quality block with low side effect profile and is sufficient to provide analgesia for major surgery at various sites with accelerated postoperative recovery. Use of Ultrasound-guided thoracic paravertebral block in the pediatric population as well as in neonates for providing perioperative analgesia is unique in itself and the literature is sparse. In our cases, we used Ultrasound-guided thoracic paravertebral block for perioperative analgesia. Usually, postoperative ventilatory support is required after TEF surgery but probably due to good lung conditions and with better analgesic technique in the form of a paravertebral block, satisfactory recovery was observed and the first two 
cases could be extubated on the same day after being shifted to the surgical intensive care unit. While in the $3 \mathrm{rd}$ neonate we were able to extubated only on the 3rd postoperative day and the reason behind could be, prior chest conditions along with associated multiple cardiac anomalies and longer duration of surgery compared to the previous two cases.

Javier et al successfully used a single shot ultrasound-guided paravertebral block with general anesthesia in 3 cases of neonates/infants for pyloromyotomy surgery as well. This technique provided adequate analgesia, avoiding the use of opioids and neuromuscular blocking agents and reducing respiratory complications associated with limiting the residual effect of opioids and the risk of central apnea allowing early extubation. ${ }^{4}$ Similarly we also used the ultrasound-guided single-shot paravertebral block as an analgesic technique for TEF repair which showed marked reduction in postoperative opioid requirement with early extubation and less postoperative pulmonary complication.

Ultrasound-guided paravertebral block practices have been reported in children. ${ }^{5,6}$ Thompson and Haynes have described their experience with successful placement and management of thoracic paravertebral block catheters in 2 neonates undergoing tracheoesophageal fistula repair via thoracotomy. Thoracic paravertebral block catheter infusions eliminated the need for a continuous sedative or narcotic infusion in their study. ${ }^{7}$ Though in our cases we only used single-shot ultrasound-guided thoracic paravertebral block as a postoperative analgesic technique which provide adequate analgesia with a significant reduction of opioid requirement in first 24 hours following surgery and added advantage of early extubation with minimal to no postoperative pulmonary complications. Placing a catheter in a paravertebral space for continuous infusion of local anaesthetic agents under sonographic guidance not only minimizes the risk of inadvertent complications, it also provides accurate placement of catheter along with adequate postoperative analgesia by significantly reducing postoperative opioids requirements. However it is always a challenge to place a catheter in a neonate but with regular practice expertise can be achieved.

\section{CONCLUSION}

We believe with the regular use of ultrasound, a sound knowledge of dermatomal distribution and sensory innervations, expertise in managing pain following thoracic surgery in neonates and children can be achieved. Adequacy of analgesia following thoracotomy for tracheoesophageal fistula repair can be achieved either with a single shot or a catheter placement technique. Ultrasound-guided thoracic Paravertebral block can be an effective, safe, and acceptable alternative following open thoracotomy in Tracheoesophageal fistula repair, providing adequate postoperative analgesia with marked reduction of opioid requirements and facilitating early extubation. However multi trials are needed to give a conclusive result.

\section{CONFLICT OF INTEREST}

\section{None}

\section{REFERENCES}

1. Spitz, L. Oesophageal atresia. Orphanet J Rare Dis 2, 24 (2007). https://doi.org/10.1186/1750-1172-2-24.

2. Bosenberg AT, Hadley GP, Wiersma R. Esophageal atresia: Caudothoracic epidural anesthesia reduces the need for post-operative ventilator support. PediatrSurgInt 1992;7:289-91. DOI:10.1111/ pan.12240.

3. Kinottenbelt G, Skinner A, Seefelder C. Tracheo-esophageal fistula(TOF) and oesophageal atresia(OA). Best Pract Res ClinAnesthesiol 2010;24:387-401.doi: 10.1016/j.bpa.2010.02.010.

4. Mata-Gómez Javier, Guerrero-DomínguezRosana, García-Santigosa Marta, Ontanilla Antonio. Ultrasound-guided paravertebral block for pyloromyotomy in 3 neonates with congenital hypertrophic pyloric stenosis. Rev. Bras. Anestesiol. 2015 Aug; 65(4): 302-305.doi: 10.1016/j.bjane.2014.03.012.
5. Boretsky K, Visoiu M, Bigeleisen P. Ultrasound-guided approach to the paravertebral space for catheter insertion in infants and children. PedAnesth 2013; 23: 1193-8 .doi: 10.1111/pan.12238.

6. Ali MA, Akbar AS. Report of a case of ultrasound guided continuous thoracic paravertebral block for post thoracotomy analgesia in a child. Middle East J Anaesthesiol 2013; 22: 107-8.doi: 10.5152/ TJAR.2016.32448.

7. Thompson ME, Haynes BRN. Ultrasound-guided thoracic paravertebral block catheter experience in 2 neonates. Journal of clinical anesthesia 2015.05.002.doi: 10.1016/j.clinane. 2015.05.002. 